\title{
Abrupt remapping in human CA3/dentate gyrus signals resolution of memory interference
}

\section{Wanjia Guo ( $\nabla$ wanjiag@uoregon.edu )}

University of Oregon https://orcid.org/0000-0002-5893-6894

\section{Serra Favila}

Columbia University https://orcid.org/0000-0003-1528-2875

\section{Ghootae Kim}

Korea Brain Research Institute

\section{Robert Molitor}

University of Oregon

\section{Brice Kuhl}

University of Oregon https://orcid.org/0000-0001-5229-5400

\section{Article}

\section{Keywords:}

Posted Date: February 12th, 2021

DOI: https://doi.org/10.21203/rs.3.rs-148842/v1

License: (c) (i) This work is licensed under a Creative Commons Attribution 4.0 International License. Read Full License

Version of Record: A version of this preprint was published at Nature Communications on August 10th, 2021. See the published version at https://doi.org/10.1038/s41467-021-25126-0. 


\section{Abrupt remapping in human $\mathrm{CA} 3 /$ dentate gyrus \\ 2 signals resolution of memory interference}

3 Wanjia Guo ${ }^{1}$, Serra E. Favila ${ }^{2}$, Ghootae $\mathrm{Kim}^{3}$, Robert J. Molitor ${ }^{1}$, Brice A. Kuhl ${ }^{1}$

4 Word Counts

5 Abstract: 150

6 Introduction, Results, Discussion: 4703

7 Methods: 3009

8 \# of Figures: 4

91 Supplementary Table

10 Keywords: hippocampus, episodic memory, pattern separation, repulsion, competition

11 Acknowledgments: This work was supported by NIH-NINDS R01 NS089729 awarded to B.A.K.

12 Author Contributions: W.G., G.K., and B.A.K. designed the experiment. W.G. and B.A.K. analyzed the 13 data. S.E.F. consulted on data analyses. All authors wrote and edited the manuscript. 


\section{ABSTRACT:}

Remapping refers to a decorrelation of hippocampal representations of similar spatial environments. While it has been speculated that remapping may contribute to the resolution of episodic memory interference in humans, direct evidence is surprisingly limited. Here, we tested this idea using high-resolution, patternbased fMRI analyses. We show that activity patterns in human CA3/dentate gyrus exhibit an abrupt, temporally-specific decorrelation of highly similar memory representations that is precisely coupled with

21 behavioral expressions of successful learning. Strikingly, the magnitude of this learning-related

22 decorrelation was predicted by the amount of pattern overlap during initial stages of learning, with greater 23 initial overlap leading to stronger decorrelation. Finally, we show that remapped activity patterns carry 24 relatively more information about learned episodic associations compared to competing associations, 25 further validating the learning-related significance of remapping. Collectively, these findings establish a 26 critical link between hippocampal remapping and episodic memory interference and provide novel insight 27 into why remapping occurs. 


\section{INTRODUCTION:}

The hippocampus is critical for forming long-term, episodic memories ${ }^{1-3}$. However, one of the fundamental challenges that the hippocampus faces is that many experiences are similar, creating the potential for memory interference ${ }^{4,5}$. In rodents, it is well established that minor alterations to the environment can trigger sudden changes in hippocampal activity patterns-a phenomenon termed remapping ${ }^{6,7}$. An appealing possibility is that hippocampal remapping also occurs in human episodic memory, allowing for similar memories to be encoded in distinct activity patterns that prevent interference ${ }^{8}$. At present, however, there remains an important gap between evidence of place cell remapping in the rodent hippocampus and episodic memory interference in humans. To bridge this gap, it is informative to consider how properties of place cell remapping, as demonstrated in the rodent hippocampus, might translate to episodic memory interference in humans.

One of the most important properties of remapping in the rodent hippocampus is that it is characterized by abrupt transitions between representations ${ }^{9-12}$. These abrupt transitions, evidenced by decorrelations in patterns of neural activity, have most typically been observed as a function of the degree of environmental change ${ }^{9,11}$. However, abrupt remapping can also occur as a function of experience with a new environment ${ }^{10,12}$. Evidence of experience-dependent remapping ${ }^{6,13}$ suggests an important point: that remapping fundamentally reflects changes in internal representations, as opposed to changes in environmental states ${ }^{14,15}$. An emphasis on internal representations lends itself well to human episodic memory in that it suggests that hippocampal remapping should occur as memories change. More specifically, this perspective makes the critical prediction that when two events are highly similar, hippocampal remapping will occur if, and when, corresponding memories become distinct. Testing this prediction requires repeatedly probing internal representations (memories) as well as hippocampal representations. However, standard approaches of averaging neuroimaging data across memories and participants can easily obscure or wash out abrupt changes in hippocampal representations if the timing of those changes varies across memories or participants.

Evidence of place cell remapping in rodents also motivates specific predictions regarding the relative contributions of hippocampal subfields, with a major distinction being between CA3/dentate gyrus and $\mathrm{CA} 1^{8,16,17}$. In general, $\mathrm{CA} 3$ and dentate gyrus are thought to be more important than $\mathrm{CA} 1$ for discriminating between similar stimuli ${ }^{15,17-20}$ and remapping has been shown to occur more abruptly in CA3 than in $\mathrm{CA} 1^{10,12,21}$. Human $\mathrm{fMRI}$ studies also support this general distinction, with several studies specifically implicating CA3 and dentate gyrus in discriminating similar memories ${ }^{22-27}$. However, these studies have not directly established a link between temporally abrupt changes in CA3/dentate gyrus activity and changes in episodic memory states.

Here, we tested whether the resolution of interference between highly similar episodic memories is associated with an abrupt remapping of activity patterns in human CA3/dentate gyrus. We used an associative memory paradigm in which participants learned and were repeatedly tested on associations between scene images and object images ${ }^{28}$. The critical design feature was that the set of scene images included pairs of extremely similar scenes (Fig. 1a). These scene pairmates were intended to elicit associative memory interference. Across six rounds of learning, we tracked improvement in associative memory for each set of pairmates while also continuously tracking representational changes indexed by fMRI. Specifically, after each associative memory test round, participants were shown each scene image one at a time (exposure phase) which allowed us to measure the activity pattern evoked by each scene and, critically, the representational distance between scene pairmates. To preview, we find that behavioral 
expressions of memory interference resolution are temporally-coupled to abrupt, stimulus-specific remapping of human $\mathrm{CA} 3 /$ dentate gyrus activity patterns. This remapping specifically exaggerated the representational distance between similar memories. In additional analyses, we show that the magnitude of remapping that individual memories experienced was predicted by the degree of initial pattern overlap among $\mathrm{CA} 3 /$ dentate gyrus representations and that remapped CA3/dentate gyrus representations carried increased and highly specific information about learned episodic associations.

\section{RESULTS:}

Participants completed six rounds of the experimental paradigm while inside an $\mathrm{fMRI}$ scanner. Each round included a study phase, an associative memory test phase, and a scene exposure phase (Fig. 1b). fMRI scanning was only conducted during the exposure phases. During the study phases, participants viewed scene-object associations one at a time. During the associative memory test phases, participants were shown scenes, one at a time, along with two very similar object choices (e.g., two guitars); one object was the target (i.e., the object that had been paired with the current scene) and the other object was the competitor (i.e., the object that had been paired with the scene pairmate). After selecting an object, participants indicated their confidence (high or low). During exposure phases, participants were shown each scene, along with novel scenes, and made a simple old/new judgment (mean $\pm 95 \% \mathrm{Cl}$ : $d^{\prime \prime}=5.40 \pm 0.88$; one-sample $t$-test vs. 0: $t_{30}=12.58, p<0.001$, Cohen's $d=2.26$ ).

\section{Behavior.}

During the associative memory test phases, participants chose the correct object with above-chance accuracy in each of the 6 rounds ( $t_{30}$ 's $\geq 2.65, p$ 's $\leq 0.013, d$ 's $\geq 0.48$; chance accuracy $=50 \%$ ). Accuracy markedly improved across rounds, from a mean of $56.45 \% \pm 4.98 \%$ in round 1 to a mean of $94.71 \% \pm 2.21 \%$ in round 6 (main effect of round: $F_{1,30}=318.86, p<0.001, \eta^{2}=0.91$ ). The rate of choosing the correct object with high-confidence also robustly increased across rounds, from a mean of $27.15 \% \pm 4.71 \%$ in

To test whether hippocampal remapping was temporally coupled with the resolution of memory interference, we identified, for each participant and for each set of pairmates, the learning round in which scene-object associations were recalled with high confidence (for both scenes in a pairmate). We refer to this timepoint as the 'learned round' (LR; see Methods). Of critical interest for our remapping analyses was the correlation of activity patterns evoked by scene images during the learned round (LR) with activity patterns evoked immediately prior to the learned round (LR-1). We refer to this transition (from pre-learned to learned) as the 'inflection point' (IP) in learning (Fig. 1d). For example, if the LR for a particular set of pairmates was round 4 , then the IP was the transition from round 3 to 4 . Our rationale for correlating activity patterns from LR-1 with LR was that this correlation would capture the critical change in hippocampal representations (remapping) that putatively supports learning. 
a

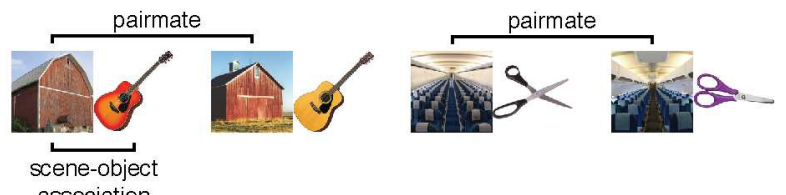

b association
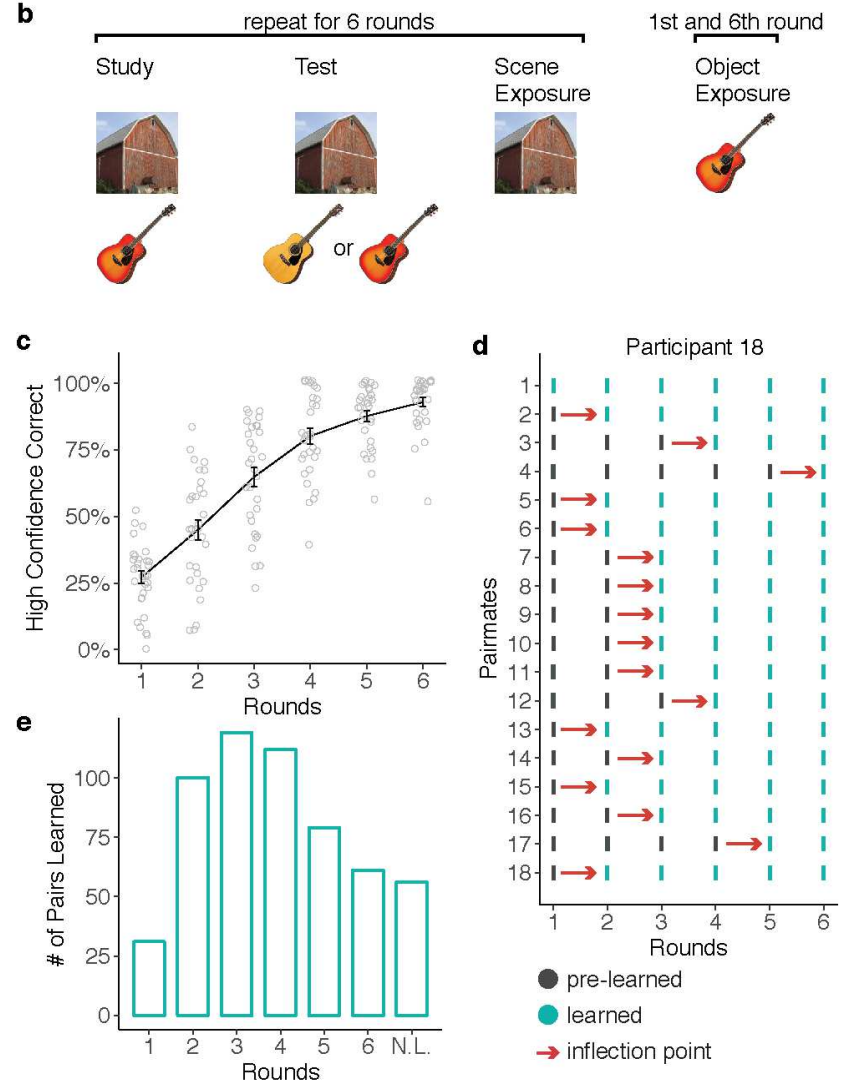

Figure 1. Experimental Design and Behavior. a. Participants learned 36 scene-object associations. The 36 scenes comprised 18 scene pairmates which consisted of highly similar image pairs (e.g., 'barn 1' and 'barn 2'). Scene pairmates were also associated with similar objects (e.g., 'guitar 1' and 'guitar 2'). b. Participants completed 6 rounds of study, test, and exposure phases. During study, participants viewed scenes and associated objects. During test, participants were presented with scenes and had to select the associated object from a set of two choices, followed by a confidence rating (high or low confidence; not shown). During exposure, scenes (rounds 1-6) or objects (round 1 and 6) were presented and participants made an old/new judgment. fMRI data were only collected during the scene and object exposure phases. c. Mean percentage of high confidence correct responses for each test round. $\mathbf{d}$. Data from a representative participant showing the 'inflection point' in learning, for each pairmate. The inflection point was defined as the point at which participants transitioned to high-confidence correct retrieval for both scenes within a pairmate $-a$ transition from 'pre-learned' to 'learned.' e. The number of pairs that transitioned to a learned state at each round, aggregated across all participants and pairmates. N.L. indicates pairmates that were never learned. Notes: error bars reflect S.E.M.

Remapping in CA3/dentate gyrus is time-locked to the inflection point in learning.

For our fMRI analyses, our primary focus was on pattern similarity between scene pairmates. Pattern similarity was measured by correlating patterns of fMRI activity evoked by each scene during the scene exposure phases. Pairmate similarity was defined as the correlation between activity patterns evoked by scene pairmates (e.g., 'barn 1' and 'barn 2'; Fig. 2b). Correlations between scenes that were not pairmates 
(e.g., 'barn 1' and 'airplane 2'; Fig. 2b) provided an important baseline measure of non-pairmate similarity. We refer to the difference between these two measures (pairmate - non-pairmate similarity) as the pairmate similarity score ${ }^{28}$. A positive pairmate similarity score would indicate that visually similar scenes (e.g., two barns) are associated with more similar neural representations than two unrelated scenes. Critically, because pairmate similarity scores are a relative measure, they can be directly compared across different brain regions ${ }^{29}$-something that would be inadvisable with raw correlation values. For all pattern similarity analyses, correlations were always performed across learning rounds (e.g., correlating 'barn 1' at LR-1 with 'barn 2' at LR). This ensured independence of $\mathrm{fMRI}$ data ${ }^{30}$, but was also intended to capture transitions in hippocampal representations (remapping).

Following a prior study that used similar stimuli and analyse ${ }^{28}$, $\mathrm{fMRI}$ analyses targeted the following regions of interest (ROIs): hippocampus, parahippocampal place area (PPA), and early visual cortex (EVC). PPA and EVC served as important control regions indexing high-level (PPA) and low-level (EVC) visual representations. We did not anticipate that these regions would demonstrate learning-related remapping. Within the hippocampus, we leveraged our high-resolution $\mathrm{fMRI}$ protocol to segment the hippocampus body into subfields comprising CA1 and CA2/CA3/dentate gyrus (CA23DG). Motivated by past empirical findings $^{23,31}$ and theoretical models ${ }^{8}$, we predicted that remapping would occur in CA23DG. More specifically, we predicted that CA23DG remapping would occur at the inflection point (IP) in learning. To test this prediction, we compared pairmate similarity scores at the IP to pairmate similarity scores at a timepoint just prior to the IP (pre-IP). Whereas pairmate similarity scores at the IP were based on correlations between activity patterns from the Learned Round (LR) and the preceding round (LR-1), pairmate similarity scores at the pre-IP were based on correlations shifted back one step in time: i.e., between LR-1 and LR-2. Thus, whereas the IP captured the transition from pre-learned to learned, the preIP was an important reference point that corresponded to a 'non-transition' (pre-learned to pre-learned).

An ANOVA with factors of behavioral state (pre-IP, IP) and ROI (CA1, CA23DG, PPA, EVC) revealed a significant main effect of $\mathrm{ROI}\left(F_{3,90}=4.08, p=0.009, \eta^{2}=0.04\right)$, reflecting overall differences in pairmate similarity scores across ROIs. Scores were numerically lowest in CA23DG and numerically highest in EVC. There was no main effect of behavioral state $\left(F_{1,30}=2.71, p=0.110, \eta^{2}=0.01\right)$, indicating that learning did not have a global effect on representational structure across ROls. Critically, however, the interaction between behavioral state and ROI was significant $\left(F_{3,90}=2.95, p=0.037, \eta^{2}=0.04\right)$, indicating that learning differentially influenced pairmate similarity scores across ROls.

Within CA23DG, pairmate similarity scores were significantly lower at the IP than the pre-IP $\left(t_{30}=-2.24, p\right.$ $=0.033, d=0.40, \mathrm{Cl}=[-0.012 \pm 0.011])$, consistent with our prediction that remapping would specifically occur at the behavioral inflection point. Importantly, we also confirmed via permutation test (see Methods) that CA23DG pairmate similarity scores at the IP were lower than would be expected if the mapping between pairmates and IP's was shuffled within participants ( $p=0.013$, one-tailed; Fig. 2 d).

Strikingly, CA23DG pairmate similarity scores not only decreased at the IP, but they were significantly below 0 at the IP $\left(t_{30}=-2.36, p=0.025, d=0.19, \mathrm{Cl}=[-0.008 \pm 0.007]\right)$. In other words, pairs of scenes with extremely high visual similarity were represented as less similar than completely unrelated scenes in CA23DG. While seemingly counterintuitive, several recent fMRI studies have also found that, in certain situations, hippocampal pattern similarity is lower for similar than dissimilar events ${ }^{23,28,32}$. This has led to 164 the proposal that similarity triggers a repulsion of hippocampal representations. That is, just as physical 165 proximity triggers repulsion of like magnetic poles, representational proximity triggers repulsion of similar 
memories (Fig. 2f). The present results, however, provide critical new evidence that this repulsion is timelocked to-and may, in fact, underlie-the resolution of interference between competing memories.

In CA1, pairmate similarity scores did not significantly differ by learning state $\left(t_{30}=-0.72, p=0.474, d=\right.$ $0.13, \mathrm{Cl}=[0.004 \pm 0.01])$ or differ from 0 either at the pre-IP $\left(t_{30}=-0.63, p=0.531, d=0.11, \mathrm{Cl}=[0.003 \pm\right.$ $0.009])$ or IP $\left(t_{30}=-0.34, p=0.735, d=0.06, \mathrm{Cl}=[-0.001 \pm 0.006]\right)$. In PPA, pairmate similarity scores decreased from pre-IP to IP $\left(t_{30}=-2.28, p=0.030, d=0.41, \mathrm{Cl}=[0.008 \pm 0.007]\right)$, with scores significantly greater than 0 in the pre-IP $\left(t_{30}=3.14, p=0.004, d=0.56, \mathrm{Cl}=[0.007 \pm 0.005]\right)$ but not different from 0 at the IP $\left(t_{30}=-0.26, p=0.798, d=0.05, \mathrm{Cl}=[-0.0006 \pm 0.005]\right)$. In EVC, pairmate similarity scores did not significantly vary by learning state $\left(t_{30}=-1.39, p=0.175, d=0.25, \mathrm{Cl}=[-0.007 \pm 0.01]\right)$; but there was a numerical increase from pre-IP to IP, with scores significantly above 0 at IP $\left(t_{30}=3.13, p=0.004, d=0.56\right.$, $\mathrm{Cl}=[0.01 \pm 0.007])$ but not at pre-IP $\left(t_{30}=0.92, p=0.366, d=0.16, \mathrm{Cl}=[0.004 \pm 0.008]\right)$.

The qualitative difference between CA23DG and EVC is striking in that, at the inflection point, these regions exhibited fully opposite representational structures: scene pairmates were more similar than non-pairmates in EVC, but less similar than non-pairmates in CA23DG. This finding parallels prior evidence of opposite representational structures in hippocampus and $\mathrm{EVC}^{28,32}$ and argues against the possibility that CA23DG 'inherited' representational structure from early visual regions. More generally, it is striking that differences in pairmate similarity scores markedly varied across the four ROls at the IP $\left(F_{3,90}=8.73, p<0.001, \eta^{2}=\right.$ $0.14)$, but not at the pre-IP $\left(F_{3,90}=0.33, p=0.804, \eta^{2}=0.008\right)$, underscoring the influence of learning on representational structure.

For the preceding fMRI analyses, the IP was defined as the correlation between the learned round (LR) and the immediately preceding round (LR-1). To more fully characterize how the representational state at the LR compared to other rounds, we additionally correlated representations at LR to representations at LR-2 and LR-3 (i.e., other rounds that preceded the LR) and also correlated LR with LR+1, LR+2, and LR+3 (rounds that followed the LR) (Fig. 2e). Within CA23DG, pairmate similarity scores were significantly lower when correlating the LR with rounds that preceded learning compared to rounds that followed learning $\left(t_{30}\right.$ $=-2.98, p=0.006, d=0.54, \mathrm{Cl}=[-0.009 \pm 0.006])$. This striking asymmetry indicates that CA23DG representations expressed at the LR were systematically biased away from the initial representational position of competing memories. More generally, these data support the idea of an abrupt representational change (remapping) in CA23DG that was time-locked to the specific round at which learning occurred for individual pairmates. For CA1, PPA, and EVC, there were no significant differences in pairmate similarity scores when correlating the LR to rounds that preceded learning vs. followed learning $\left(\left|t_{30}\right| \leq 0.79, p\right.$ 's $\geq$ $0.435, d \leq 0.14$; Fig. 2e). 
a

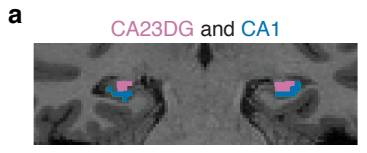

$\mathbf{C}$

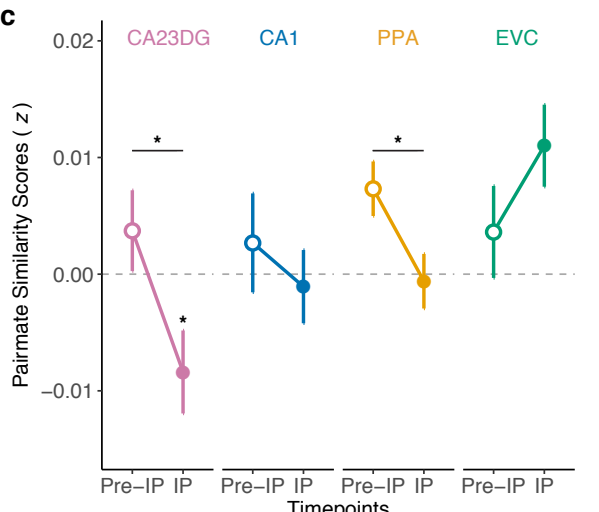

e

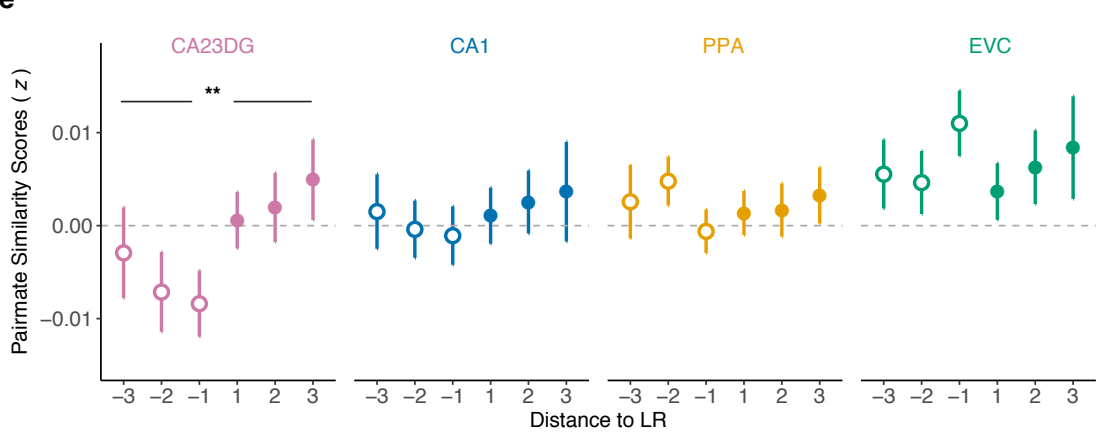

b

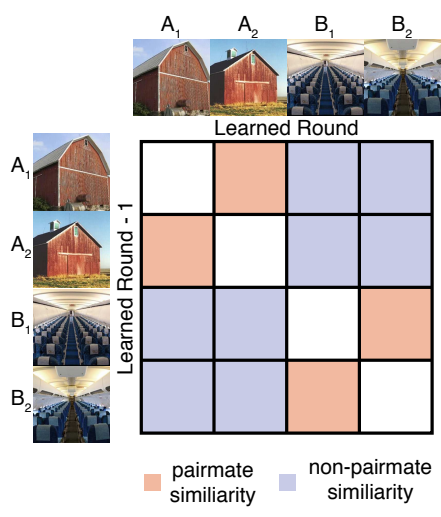

Pairmate Similiarty Scores $=$

pairmate similiarity - non-pairmate similiarity

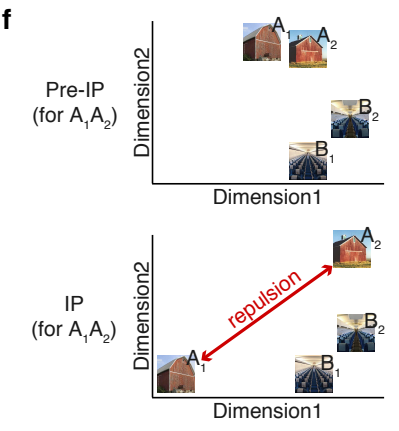

Figure 2. Pairmate similarity scores change at the behavioral inflection point. a. Regions of interest included CA23DG and CA1 in the hippocampus, the parahippocampal place area (PPA), and early visual cortex (EVC). b. Correlation matrix illustrating how pairmate similarity scores were computed for the behavioral inflection point. c. Pairmate similarity scores at the behavioral inflection point (IP) and just prior to the inflection point (pre-IP) across different regions of interest (ROIs). Pairmate similarity scores significantly varied by $\mathrm{ROI}(p=0.009)$ and there was a significant interaction between ROls and behavioral state $(p=0.011)$. d. A permutation test $(1,000$ iterations $)$ was performed by shuffling, within participants, the mapping between the behavioral inflection point and scene pairmates. In CA23DG the actual mean group-level pairmate similarity score at the IP was lower than $98.70 \%$ of the permuted mean similarity scores. e. Pairmate similarity scores calculated by correlating the learned round (LR) with each of the three preceding rounds (- distance to LR) and each of the three succeeding rounds (+ distance to LR). In CA23DG, pairmate similarity scores were significantly lower when the LR was correlated with preceding round compared to succeeding rounds $(p=0.006)$. The difference was not significant for any other ROIs ( $p$ 's $\geq 0.435)$. f. Conceptual illustration of a decrease in pairmate similarity scores from pre-IP to IP. In the pre-IP state (top panel), $A_{1}$ and $A_{2}$ are nearby in representational space. In the IP state (bottom panel), the representational distance between $A_{1}$ and $A_{2}$ has been exaggerated. When pairmates (e.g., $A_{1}$ and $A_{2}$ ) are farther apart in representational space than non-pairmates (e.g., $A_{1}$ and $B_{2}$ ) the pairmate similarity score will be negative (i.e., pairmate similarity < non-pairmate similarity), consistent with a repulsion of competing representations. Notes: ${ }^{\star} p<.05,{ }^{\star \star} p<.01$, error bars reflect S.E.M.

Overlap of CA23DG representations triggers remapping.

The fact that pairmate similarity scores in CA23DG were negative at the IP (Fig. 2c) suggests that learningrelated remapping involved an active repulsion of competing hippocampal representations (Fig. 2f). Conceptually, the key feature of a repulsion account is that separation of hippocampal representations is a reaction to initial overlap among memories ${ }^{33}$. Here, because we measured representational states throughout the course of learning, we were able to test this hypothesis directly. Specifically, we tested the 
prediction that relatively greater pairmate similarity scores (i.e., higher overlap between memories) at a given timepoint is associated with relatively lower pairmate similarity scores (i.e., lower overlap between memories) at a successive timepoint.

To test this hypothesis, we first translated the 6 learning rounds into 5 'timepoints' (see Methods). Each timepoint corresponded to the set of scene pair similarity scores obtained by correlating activity patterns across consecutive learning rounds [e.g., timepoint $1=r($ round 1 , round 2)]. These scores reflected the representational structure at each timepoint (i.e., which pairmates were relatively similar, which pairmates were relatively dissimilar). We then rank correlated the pairmate similarity scores across successive timepoints [r(timepoint 1, timepoint 2)]. Whereas a positive rank correlation would indicate that representational structure is preserved across time points, a negative rank correlation would indicate that representational structure is inverted across time points. Critically, an inversion of representational structure is precisely what would be predicted if initial overlap among activity patterns (i.e., high pairmate similarity scores) triggers a repulsion of activity patterns (i.e., low pairmate similarity scores).

Strikingly, the rank correlation in CA23DG was significantly negative $\left(t_{30}=-2.99, p=0.006, d=0.54, \mathrm{Cl}=\right.$ $[-0.06 \pm 0.04])$. In contrast, the rank correlation in CA1 was significantly positive $\left(t_{30}=2.11, p=0.043, d=\right.$ $0.38, \mathrm{Cl}=[0.06 \pm 0.05])$. The difference between CA23DG and CA1 was also significant $\left(t_{30}=3.73, p<\right.$ $0.001, d=0.67, \mathrm{Cl}=[0.12 \pm 0.06])$. Importantly, the negative correlation in CA23DG cannot be explained by regression to the mean (see Methods). Moreover, when we tested correlations at a lag of 2 [ $r$ (timepoint $\mathrm{N}$, timepoint $\mathrm{N}+2)]$, correlations did not significantly differ from 0 for either CA23DG $\left(t_{30}=-0.71, p=0.485\right.$, $d=0.13, \mathrm{Cl}=[-0.02 \pm 0.05])$ or $\mathrm{CA} 1\left(t_{30}=-1.60, p=0.120, d=0.29, \mathrm{Cl}=[-0.04 \pm 0.05]\right)$. Further, the interaction between lag $(1,2)$ and ROI (CA23DG, CA1) was also significant $\left(F_{1,30}=7.09, p=0.012, \eta^{2}=\right.$ 0.06 ), indicating that the dissociation between CA23DG and CA1 was relatively stronger at lag 1 (consecutive timepoints) than lag 2 (non-consecutive timepoints). Thus, representational structure at a given time point specifically predicted representational structure at a successive timepoint. Rank correlations did not differ from 0 in either PPA or EVC, either for lag 1 or lag 2 ( $\left|t_{30}\right|$ 's $\leq 1.12, p$ 's $\geq 0.272$, $\left.d^{\prime} \mathrm{s} \leq 0.20\right)$.

While the negative correlation in CA23DG was fully consistent with our prediction-and with the idea that high pattern overlap triggers repulsion-the negative correlation could alternatively be explained by pairmates with relatively low pairmate similarity at timepoint $N$ tending to have relatively high similarity at timepoint $\mathrm{N}+1$. Additionally, because our analysis was entirely agnostic to behavioral data, it does not specifically establish that the negative pairmate similarity scores that we observed at the behavioral IP (Fig. 2c and $\mathbf{2 e}$ ) were triggered by pattern overlap at IP-1. Thus, as a complementary analysis, we binned all pairmates, by quartiles, according to pairmate similarity scores at IP-1, with the $4^{\text {th }}$ quartile representing pairmates with the highest pairmate similarity scores. We then computed the mean pairmate similarity scores for those bins at the IP. Again, this analysis was separately performed for CA23DG and CA1. An ANOVA with factors of ROI (CA23DG, CA1) and pairmate similarity scores at IP-1 (4 quartiles) revealed a significant interaction $\left(F_{3,90}=3.19, p=0.027, \eta^{2}=0.03\right)$. Critically, this interaction was driven by a marked difference between CA23DG and CA1 when considering the bin with the highest overlap at IP-1 (i.e., 4th quartile: $t_{30}=-2.87, p=0.008, d=0.51, \mathrm{Cl}=[-0.03 \pm 0.02]$, Fig. 3c). For CA23DG, pairmate similarity scores at the IP were significantly below 0 and numerically lowest for pairmates whose similarity scores at $\mathrm{IP}-1$ were in the $4^{\text {th }}$ quartile (comparison to $0: t_{30}=-2.54, p=0.017, d=0.46, \mathrm{Cl}=[-0.023 \pm 0.019]$ ); the pattern in CA1 was qualitatively opposite. Collectively, these results provide novel, theory-consistent evidence that remapping of competing representations is actively triggered by initial representational overlap. 
a

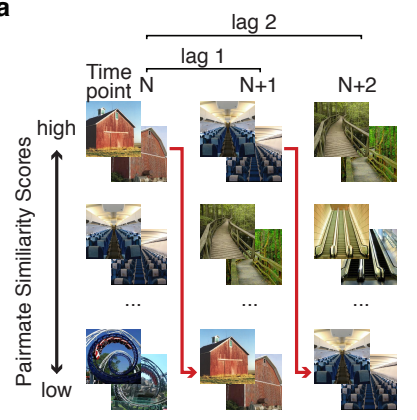

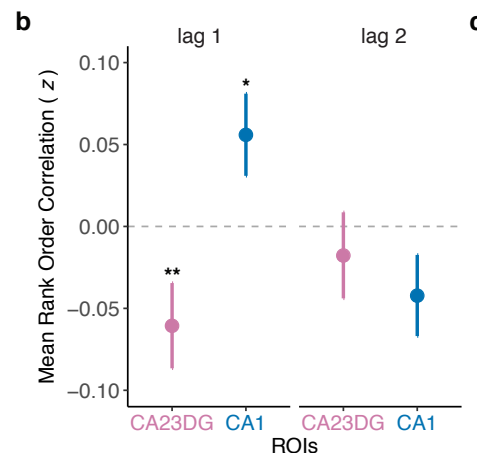

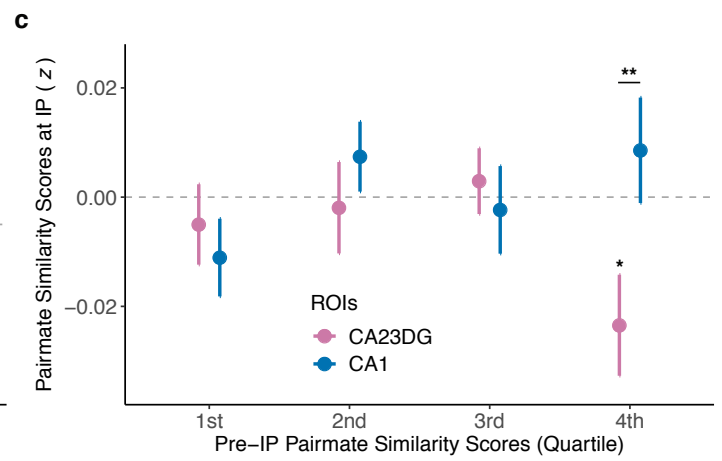

Figure 3. Representational structure across timepoints. a. Schematic illustration showing the rank order of scene pairmates based on pairmate similarity scores at various time points $(\mathrm{N}, \mathrm{N}+1, \mathrm{~N}+2)$. If scene pairmates with relatively high pairmate similarity scores at a given timepoint are systematically associated with relatively low pairmate similarity scores at a succeeding time point (red arrows), this will produce a negative rank correlation. b. Mean rank order correlations of pairmate similarity scores across timepoints for CA23DG and CA1. Lag 1 correlations reflect correlations between a given timepoint and an immediate succeeding timepoint (e.g., timepoints 2 and 3). Lag 2 correlations reflect correlations between a given timepoint and a timepoint two steps away (e.g., timepoints 2 and 4). At lag 1, there was a negative correlation in CA23DG $(p=0.004)$, but a positive correlation in CA1 $(p=0.045)$. At lag2, correlations were not significant in either CA23DG or CA1 indicating that correlations in representational structure were specific to temporally adjacent rounds. c. Pairmate similarity scores at the inflection point (IP) as a function of relative pairmate similarity scores in the pre-IP state $\left(1^{\text {st }}\right.$ quartile $=$ lowest similarity, $4^{\text {th }}$ quartile $=$ highest similarity $)$. Pairmate similarity scores in CA23DG were significantly lower than CA1 $(p=0.017)$ and significantly below $0(p=0.008)$ for pairmates with the highest pre-IP similarity (4th quartile). Notes: ${ }^{*} p<.05,{ }^{* *} p<.01$, error bars reflect S.E.M.

\section{CA23DG scene representations differentiate between competing object associations.}

Thus far, we have focused on similarity among neural representations evoked while viewing the scene images (scene exposure phase). However, our paradigm also included two fMRI runs during which participants viewed each of the objects associated with the scene images (object exposure phase; see Methods). This allowed us to test whether hippocampal activity patterns evoked while viewing the scenes resembled-or came to resemble-activity patterns evoked while viewing corresponding object images.

Whereas, pairmate similarity scores were computed by correlating activity patterns across rounds of the scene exposure phase (e.g., LR-1 and LR), here we computed correlations between a single round of the scene exposure phase (e.g., LR) and the average of the two object rounds (see Methods). For this analysis, there were three important factors that we considered. First, we considered whether scene representations were in a 'pre-learned' state (LR-1) or 'learned' state (LR). Second, we separately tested correlations between each scene and (a) the target object (e.g., 'guitar 1') vs. (b) the competing object (e.g., 'guitar 2') (Fig. 4a). Third, we again compared results in CA23DG vs. CA1.

A repeated measures ANOVA with factors of ROI (CA23DG, CA1), behavioral state (pre-learned, learned), and object relevance (target, competitor) revealed a significant interaction between behavioral state and

273 object relevance $\left(F_{1,30}=12.42, p=0.001, \eta^{2}=0.02\right)$. Qualitatively, this interaction reflected a learning274 related change wherein hippocampal representations of scene images became relatively more similar to 
target objects and less similar to competitor objects. However, this 2-way interaction between behavioral state and object relevance was qualified by a trend toward a 3-way interaction between behavioral state, object relevance, and $\mathrm{ROI}\left(F_{1,30}=4.07, p=0.053, \eta^{2}=0.01\right)$. Specifically, the interaction between behavioral state (pre-learned, learned) and object relevance (target, competitor) was significant in CA23DG $\left(F_{1,30}=11.98, p=0.002, \eta^{2}=0.06\right)$ but not in CA1 $\left(F_{1,30}=0.44, p=0.510, \eta^{2}=0.002\right)$ (Fig. 4b). For CA23DG, there was a qualitative increase, from the pre-learned to learned state, in similarity between scenes and target objects and a qualitative decrease, from the pre-learned to learned state, in similarity between scenes and competing objects. In other words, the remapping of CA23DG scene representations that occurred at the learned round yielded a relative strengthening of information related to target object associations and a relative weakening of information related to competing object associations. This dissociation in CA23DG is striking when considering that target and competitor objects were extremely similar (see Fig.1a, Fig. 4a) and even more so when considering that during the scene and object exposure phases participants were not instructed or required in any way to recall the corresponding images. The 2way interaction between behavioral state and object relevance was not significant for PPA or EVC [ $F_{1,30}$ 's $\left.\leq 3.23, p^{\prime} s \geq 0.082, \eta^{2} s \leq 0.02\right]$.

a

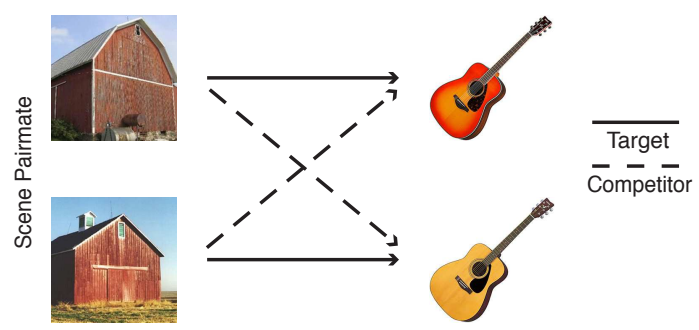

b

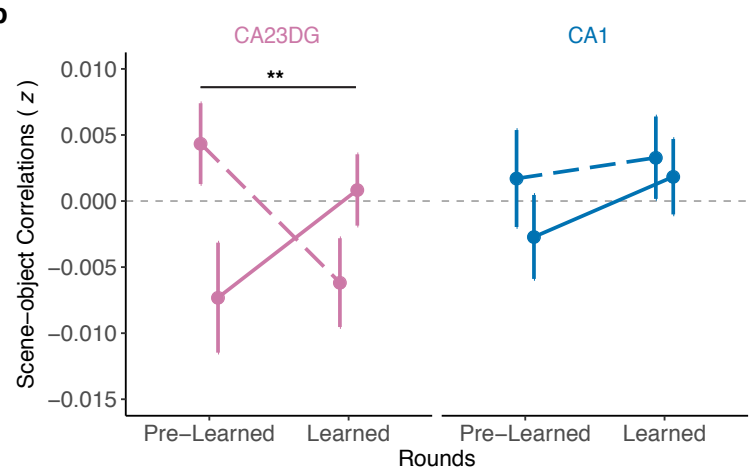

Figure 4. Scene-object similarity as a function of behavioral state. a. Example associations between scene pairmates and objects. Scene-object similarity was calculated by correlating activity patterns evoked during the scene exposure phases (at different behavioral states) and the object exposure phases. Target similarity refers to correlations between a given scene and the object with which it was studied. Competitor similarity refers to correlations between a given scene and the object with which its pairmate was studied. b. Scene-object similarity as a function of object relevance (target, competitor), ROI (CA23DG, CA1), and behavioral state (pre-learned, learned). Correlations between unrelated scenes and objects (across pairmate similarity; not shown) was subtracted from target and competitor similarity values. For CA23DG, there was a significant interaction between behavioral state and object relevance $(p=$ 0.002). Notes: ${ }^{\star \star} p<.01$, error bars reflect S.E.M. 

Here, we show that learning to discriminate competing episodic memories is associated with an abrupt remapping of activity patterns in CA3/dentate gyrus. Specifically, fMRI pattern similarity in CA3/dentate gyrus decreased precisely when behavioral expressions of learning emerged. Additionally, the degree to which remapping occurred in CA3/dentate gyrus was predicted by the degree of initial pattern overlap among competing memories. Finally, remapped CA3/dentate gyrus representations contained relatively stronger information about relevant episodic associations and relatively weaker information about competing episodic associations, confirming the learning-related significance of the remapping effect.

Our findings complement recent demonstrations of remapping-like phenomena in the human hippocampus ${ }^{34,35}$ as well as evidence of abrupt remapping in the rodent hippocampus ${ }^{9-12}$. However, our findings provide unique and direct support for the proposal that hippocampal remapping is associated with the resolution of human episodic memory interference ${ }^{8}$. Specifically, we demonstrate an abrupt transition in hippocampal representations that occurred at an important inflection point in learning - the point at which participants were able to correctly discriminate similar memories and retrieve associations with high confidence. Notably, this finding was only possible because (a) we repeatedly probed episodic memory and hippocampal representations over the course of learning and (b) we identified inflection points in a participant- and pairmate-specific manner. Indeed, inflection points varied considerably across and within participants (Fig. 1d and Sup. table 1) and the observed hippocampal remapping effect was significantly weaker when the specific mapping between behavior and fMRI data was shuffled within participants (Fig. 2d).

The fact that CA23DG remapping occurred precisely at the inflection point in learning strongly suggests that remapping was related to learning. This argument is also reinforced by our independent finding that remapped CA23DG activity patterns, evoked while participants viewed individual scene images, carried more information (compared to the pre-learning state) about target versus competing object associations. In other words, the inflection point defined from behavioral expressions of associative memory also captured a critical change in associative representations encoded in CA23DG activity patterns. The fact that CA23DG exaggerated the representational distance between competing scenes (remapping) while simultaneously reflecting learned associations (scene-object similarity) is consistent with the idea that CA3 balances both pattern separation and pattern completion mechanisms ${ }^{4,17,36,37}$. The fact that remapped activity patterns contained information about learned associations is also consistent with the argument that hippocampal remapping does not simply reflect changes in the external environment-which did not change over the course of the experiment-but instead fundamentally reflects changes in internal models of the environment ${ }^{14,15}$.

One aspect of our findings which does not, to our knowledge, have a direct analog in rodent studies of remapping is the negative pairmate similarity score we observed at the inflection point in CA23DG. The negative score indicates that scene pairmates-which were extremely similar images-were associated with less overlapping CA23DG representations than completely unrelated scenes. In rodents, the most extreme version of remapping occurs when two similar environments are associated with fully independent place $\operatorname{codes}^{8}$. In our study, however, if each scene was associated with an independent representation, then the similarity between pairmates would be equal to, but not lower than, the similarity between nonpairmates. Instead, the negative pairmate similarity score requires a dependence between competing hippocampal representations wherein a given memory representation systematically moves away from the representational position of a competing memory (Fig. 2f). We refer to this dependence as 'repulsion' in 
order to emphasize the oppositional influence that competing memories exerted. Several recent human fMRI studies have reported conceptually similar effects in the hippocampus ${ }^{28,32,38}$-and in CA3/dentate gyrus, specifically ${ }^{22-26}$. However, the current findings are the first to directly establish that the repulsion of competing hippocampal representations is temporally coupled to the resolution of memory interference.

Based on computational models ${ }^{33,39,40}$, our prediction was that the repulsion effect in CA23DG was a direct consequence of initial overlap among activity patterns. Indeed, a recent study found that hippocampal repulsion was more likely to occur for behaviorally-confusable memories ${ }^{32}$, potentially because confusable memories are associated with greater pattern overlap during initial learning. In the current study, we tested-and confirmed-this account directly. Specifically, we found that the representational structure (relative pairmate similarity) in CA23DG at a given timepoint was negatively correlated with representational structure at an immediately following timepoint. This negative relationship is highly consistent with the idea that overlap, itself, triggers plasticity that 'punishes' those features which are shared across memories ${ }^{24,33,39,40}$. While our study does not afford inferences about the causal relationship between repulsion and learning, the idea that repulsion (or remapping more generally) is triggered by representational overlap, combined with the fact that remapping was associated with learning, is consistent with the possibility that repulsion of $\mathrm{CA} 3 /$ dentate gyrus representations is a causal factor in learning.

Across multiple analyses, we observed dissociations between CA3/dentate gyrus and CA1. The fact that the remapping effects were selective to $C A 3 /$ dentate gyrus is consistent with evidence from rodent studies of remapping and pattern separation ${ }^{8,16,36}$ and with several human fMRI studies ${ }^{22-25,36}$. Perhaps the most striking dissociation between CA23DG and CA1 comes from our analysis of representational structure across time points. Whereas CA23DG exhibited a negative rank correlation across successive timepoints, CA1 exhibited a positive rank correlation (Fig. 3b). Thus, in contrast to CA23DG, CA1 was characterized by stability (though only modest stability) of representational structure across timepoints ${ }^{4}$. This dissociation between CA23DG and CA1 is consistent with the idea that CA3, in particular, supports rapid plasticity that allows for changes in memory representations on short time scales ${ }^{41}$ and is also consistent with evidence of faster remapping in $C A 3 /$ dentate gyrus than in $C A 1^{10,12,21}$. It is also notable that the remapping effect we observed in CA23DG at the inflection point in learning strongly contrasted with the pattern of data in early visual cortex. Whereas CA23DG exhibited a negative pairmate similarity score at the inflection point, EVC exhibited a significant, positive pairmate similarity score at the inflection point. This finding makes the important point that CA23DG was not inheriting representational structure from early sensory regions (e.g., due to visual attention)-rather, CA23DG fully inverted the representational structure that was expressed in early visual cortex ${ }^{28}$.

Taken together, our findings constitute novel evidence for a remapping of human CA3/dentate gyrus representations that is temporally-coupled to the resolution of episodic memory interference. These findings were motivated by-and complement-existing evidence of remapping in the rodent hippocampus. Yet, our findings also go beyond existing rodent or human studies by establishing a direct link between remapping and changes in internal memory states ${ }^{14,15}$. Additionally, our conclusion that overlap among CA3/dentate gyrus representations actively triggers a repulsion of memory representations has important implications for theoretical accounts of how the hippocampus resolves memory interference ${ }^{5,8,36,39}$ and will hopefully inspire targeted new analyses that test for similar mechanisms in rodent models. 
REFERENCES:

381 1. Eichenbaum, H. A cortical-hippocampal system for declarative memory. Nat. Rev. Neurosci. 1, 41-50 382 (2000).

383 2. Squire, L. \& Zola-Morgan, S. The medial temporal lobe memory system. Science 253, 1380-1386 384 (1991).

3. O’Keefe, J. \& Nadel, L. The hippocampus as a cognitive map. (Clarendon Press; Oxford University Press, 1978).

4. Schapiro, A. C., Turk-Browne, N. B., Botvinick, M. M. \& Norman, K. A. Complementary learning systems within the hippocampus: a neural network modelling approach to reconciling episodic memory with statistical learning. Philos. Trans. R. Soc. B Biol. Sci. 372, 20160049 (2017).

5. O'Reilly, R. C. \& Norman, K. A. Hippocampal and neocortical contributions to memory: advances in the complementary learning systems framework. Trends Cogn. Sci. 6, 505-510 (2002).

6. Bostock, E., Muller, R. U. \& Kubie, J. L. Experience-dependent modifications of hippocampal place cell firing. Hippocampus 1, 193-205 (1991).

7. Muller, R. U. \& Kubie, J. L. The effects of changes in the environment on the spatial firing of hippocampal complex-spike cells. J. Neurosci. Off. J. Soc. Neurosci. 7, 1951-1968 (1987).

8. Colgin, L. L., Moser, E. I. \& Moser, M.-B. Understanding memory through hippocampal remapping.

$397 \quad$ Trends Neurosci. 31, 469-477 (2008).

398 9. Colgin, L. L. et al. Attractor-Map Versus Autoassociation Based Attractor Dynamics in the 399 Hippocampal Network. J. Neurophysiol. 104, 35-50 (2010).

400 10. Leutgeb, S., Leutgeb, J. K., Moser, E. I. \& Moser, M.-B. Fast rate coding in hippocampal CA3 cell 401 ensembles. Hippocampus 16, 765-774 (2006).

402 Wills, T. J. Attractor Dynamics in the Hippocampal Representation of the Local Environment. $403 \quad$ Science 308, 873-876 (2005).

404 12. Lee, I., Rao, G. \& Knierim, J. J. A Double Dissociation between Hippocampal Subfields: 405 Differential Time Course of CA3 and CA1 Place Cells for Processing Changed Environments. Neuron $406 \quad$ 42, 803-815 (2004). 
407 13. Lever, C., Wills, T., Cacucci, F., Burgess, N. \& O’Keefe, J. Long-term plasticity in hippocampal 408 place-cell representation of environmental geometry. Nature 416, 90-94 (2002).

409 14. Sanders, H., Wilson, M. A. \& Gershman, S. J. Hippocampal remapping as hidden state inference. $410 \quad$ eLife 9, e51140 (2020).

411 15. Keinath, A. T., Nieto-Posadas, A., Robinson, J. C. \& Brandon, M. P. DG-CA3 circuitry mediates 412 hippocampal representations of latent information. Nat. Commun. 11, 3026 (2020).

413 16. Duncan, K. D. \& Schlichting, M. L. Hippocampal representations as a function of time, subregion, 414 and brain state. Neurobiol. Learn. Mem. 153, 40-56 (2018).

415 17. Guzowski, J. F., Knierim, J. J. \& Moser, E. I. Ensemble Dynamics of Hippocampal Regions CA3 $416 \quad$ and CA1. Neuron 44, 581-584 (2004).

417 18. McHugh, T. J. et al. Dentate Gyrus NMDA Receptors Mediate Rapid Pattern Separation in the $418 \quad$ Hippocampal Network. Science 317, 94-99 (2007).

419 19. Leutgeb, S., Leutgeb, J. K., Treves, A., Moser, M.-B. \& Moser, E. I. Distinct Ensemble Codes in 420 Hippocampal Areas CA3 and CA1. Science 305, 1295-1298 (2004).

421 20. Vazdarjanova, A. \& Guzowski, J. F. Differences in Hippocampal Neuronal Population Responses 422 to Modifications of an Environmental Context: Evidence for Distinct, Yet Complementary, Functions of 423 CA3 and CA1 Ensembles. J. Neurosci. 24, 6489-6496 (2004).

424 21. van Dijk, M. T. \& Fenton, A. A. On How the Dentate Gyrus Contributes to Memory Discrimination. $425 \quad$ Neuron $98,832-845 . e 5$ (2018).

$42622 . \quad$ Molitor, R. J., Sherrill, K. R., Morton, N. W., Miller, A. A. \& Preston, A. R. Memory reactivation 427 during learning simultaneously promotes dentate gyrus/CA2,3 pattern differentiation and CA1 memory 428 integration. J. Neurosci. (2020) doi:10.1523/JNEUROSCI.0394-20.2020.

429 23. Dimsdale-Zucker, H. R., Ritchey, M., Ekstrom, A. D., Yonelinas, A. P. \& Ranganath, C. CA1 and 430 CA3 differentially support spontaneous retrieval of episodic contexts within human hippocampal $431 \quad$ subfields. Nat. Commun. 9, 294 (2018).

432 24. Kim, G., Norman, K. A. \& Turk-Browne, N. B. Neural Differentiation of Incorrectly Predicted 433 Memories. J. Neurosci. 37, 2022-2031 (2017). 
434 25. Copara, M. S. et al. Complementary Roles of Human Hippocampal Subregions during Retrieval 435 of Spatiotemporal Context. J. Neurosci. 34, 6834-6842 (2014).

436 26. Schapiro, A. C., Kustner, L. V. \& Turk-Browne, N. B. Shaping of Object Representations in the 437 Human Medial Temporal Lobe Based on Temporal Regularities. Curr. Biol. 22, 1622-1627 (2012).

438 27. Bakker, A., Kirwan, C. B., Miller, M. \& Stark, C. E. L. Pattern separation in the human 439 hippocampal CA3 and dentate gyrus. Science 319, 1640-1642 (2008).

440 28. Favila, S. E., Chanales, A. J. H. \& Kuhl, B. A. Experience-dependent hippocampal pattern 441 differentiation prevents interference during subsequent learning. Nat. Commun. 7, 11066 (2016).

442 29. Kriegeskorte, N. Representational similarity analysis - connecting the branches of systems 443 neuroscience. Front. Syst. Neurosci. (2008) doi:10.3389/neuro.06.004.2008.

444 30. Mumford, J. A., Davis, T. \& Poldrack, R. A. The impact of study design on pattern estimation for 445 single-trial multivariate pattern analysis. Neurolmage 103, 130-138 (2014).

446 31. Leutgeb, J. K., Leutgeb, S., Moser, M.-B. \& Moser, E. I. Pattern Separation in the Dentate Gyrus 447 and CA3 of the Hippocampus. Science 315, 961-966 (2007).

448 32. Chanales, A. J. H., Oza, A., Favila, S. E. \& Kuhl, B. A. Overlap among Spatial Memories Triggers 449 Repulsion of Hippocampal Representations. Curr. Biol. 27, 2307-2317.e5 (2017).

450 33. Hulbert, J. C. \& Norman, K. A. Neural Differentiation Tracks Improved Recall of Competing 451 Memories Following Interleaved Study and Retrieval Practice. Cereb. Cortex 25, 3994-4008 (2015).

452 34. Kyle, C. T., Stokes, J. D., Lieberman, J. S., Hassan, A. S. \& Ekstrom, A. D. Successful retrieval of 453 competing spatial environments in humans involves hippocampal pattern separation mechanisms. $454 \quad$ elife 4, e10499 (2015).

455 35. Steemers, B. et al. Hippocampal Attractor Dynamics Predict Memory-Based Decision Making. 456 Curr. Biol. 26, 1750-1757 (2016).

457 36. Yassa, M. A. \& Stark, C. E. L. Pattern separation in the hippocampus. Trends Neurosci. 34, 515$458 \quad 525(2011)$

459 37. Hindy, N. C., Ng, F. Y. \& Turk-Browne, N. B. Linking pattern completion in the hippocampus to 460 predictive coding in visual cortex. Nat. Neurosci. 19, 665-667 (2016). 
461 38. Jiang, J., Wang, S.-F., Guo, W., Fernandez, C. \& Wagner, A. D. Prefrontal reinstatement of 462 contextual task demand is predicted by separable hippocampal patterns. Nat. Commun. 11, 2053 463 (2020).

464 39. Ritvo, V. J. H., Turk-Browne, N. B. \& Norman, K. A. Nonmonotonic Plasticity: How Memory 465 Retrieval Drives Learning. Trends Cogn. Sci. 23, 726-742 (2019).

466 40. Norman, K. A., Newman, E. L. \& Detre, G. A neural network model of retrieval-induced forgetting. 467 Psychol. Rev. 114, 887-953 (2007).

468 41. Rebola, N., Carta, M. \& Mulle, C. Operation and plasticity of hippocampal CA3 circuits:

469 implications for memory encoding. Nat. Rev. Neurosci. 18, 208-220 (2017).

470 


\section{Participants.}

474 Thirty-six participants (21 female; mean age $=23.69 \mathrm{yrs}$, range $=18-34 \mathrm{yrs}$ ) were enrolled in the 475 experiment following procedures approved by the University of Oregon Institutional Review Board. All participants were right-handed native-English speakers with normal or corrected-to-normal vision, with no self-reported psychiatric or neurological disease. One participant was excluded due to excess motion in the scanner (max FD > $3.5 \mathrm{~mm}$ ); another 4 participants were excluded due to low behavioral performance (see Results for more details). The final analysis included 31 participants. All participants received monetary compensation for participating.

\section{Stimuli.}

Thirty-six images of scenes and 36 images of everyday objects were used in the experiment. The set of 36 scenes and the set of 36 objects were each comprised of 18 'pairmates' of visually and semantically similar images (Fig. 1a). An additional 36 scenes and 12 objects were used as lures for the scene and object exposure phases of the study, respectively. Separately for each participant, scene pairmates were randomly assigned to object pairmates (Fig. 1a). For example, if 'barn 1' was assigned to 'guitar 1', then 'barn 2' would be assigned to 'guitar 2.'

\section{Experimental procedure.}

After providing consent and reviewing the instructions, participants entered the MRI scanner. Inside the scanner, participants completed 6 rounds of the experimental paradigm (Fig. 1b). The first round and the last round included 4 phases: study, test, scene exposure (scanned), and object exposure (scanned). Rounds 2-5 were the same, except they did not include the object exposure phase. Across all phases, stimuli were displayed on a grey background, projected from the back of the scanner. After exiting the scanner, participants completed a separate memory task that involved learning new scene-object associations (not reported here). The experiment was implemented in $\mathrm{PsychoPy}^{1}$ and lasted approximately $3 \mathrm{hrs}$, with about 2 hrs 15 min inside the scanner.

Study Phase. During the study phases, participants learned 36 scene-object associations, one association at a time. Each trial began with the presentation of a scene image $(1000 \mathrm{~ms})$, followed by a white fixation cross $(200 \mathrm{~ms})$, the associated object image $(1000 \mathrm{~ms})$ and then another white fixation cross (1200 ms) until the start of the next trial. The order in which the 36 scene-object associations were studied was randomized for each round and for each participant.

Test Phase. During the test phases, participants attempted to retrieve the object associated with each of the 36 scenes. Each trial began with the presentation of a scene $(1000 \mathrm{~ms})$, followed by a white fixation cross (200 ms), and then the presentation of two object pairmates (e.g., 'Guitar 1' and 'Guitar 2'). One of the object images was the 'target' (i.e., the object associated with the cued scene) and the other object image was the 'competitor' (i.e., the object associated with the cued scene's pairmate). Participants had a maximum of $4000 \mathrm{~ms}$ to select the correct object image (target) via a button box in their right hand. If no response was made, the next trial began after a white fixation cross was displayed for $1200 \mathrm{~ms}$. If a response was made, a confidence rating then appeared beneath the objects and participants had a maximum of $3000 \mathrm{~ms}$ to indicate whether their response was a "Guess" or "Sure." After indicating their confidence (or after time ran out), a white fixation cross appeared (1200 ms) until the start of the next trial. The location of the correct object (left or right) and the order in which each of the 36 scene-object associations were tested were randomized for each round and for each participant. 
Scene Exposure Phase. During the scene exposure phases, which were conducted during $\mathrm{fMRI}$ scanning, participants saw 39 scene images in each of two blocks (78 scenes per round). Each block included the 36 studied scenes and 3 novel lure scenes. Participants made an old/new judgment for each scene. Each trial began with the presentation of a scene image $(500 \mathrm{~ms})$, followed by a red fixation cross $(1500 \mathrm{~ms})$ which represented the response window. Participants again responded using the button box. After the red fixation cross, a white fixation cross $(2000 \mathrm{~ms})$ was presented until the start of the next trial. The order of the 39 scene trials within each block was randomized for each block, round, and participant. Between the two blocks of 39 trials, participants performed a short odd/even judgment task (4 trials). Each odd/even trial consisted of a single-digit number displayed on the screen $(500 \mathrm{~ms})$, followed by a red fixation cross (1000 $\mathrm{ms}$ ) which represented the response window, and then a white fixation cross (1000 ms) until the start of the next trial.

Object Exposure Phase. The object exposure phase (conducted during fMRI scanning) was only included in the first and sixth rounds and followed an identical structure and procedure as the scene exposure phase. The only difference was that the 39 trials in each block corresponded to the 36 studied objects and 3 novel lure objects.

\section{MRI acquisition.}

All images were acquired on a Siemens 3T Skyra MRI system in the Lewis Center for Neuroimaging at the University of Oregon. Functional data were acquired with a T2*-weighted echo-planar imaging sequence with partial-brain coverage that prioritized full coverage of the hippocampus and early visual cortex (repetition time $=2000 \mathrm{~ms}$, echo time $=36 \mathrm{~ms}$, flip angle $=90^{\circ}, 72$ slices, $1.7 \times 1.7 \times 1.7 \mathrm{~mm}$ voxels). A total of 8 functional scans were acquired. Each functional scan comprised 177 volumes and included $10 \mathrm{~s}$ of lead-in time and $10 \mathrm{~s}$ of lead-out time at the beginning and end of each scan, respectively. The 8 functional scans corresponded to 6 rounds of the scene exposure phase (scans 1 and 3-7) and 2 rounds of the object exposure phase (scans 2 and 8). Anatomical scans included a whole-brain high-resolution T1-weighted magnetization prepared rapid acquisition gradient echo anatomical volume (1x1x1mm voxels) and a highresolution (coronal direction) T2-weighted scan $(0.43 \times 0.43 \times 2 \mathrm{~mm}$ voxels) to facilitate segmentation of hippocampal subfields.

\section{Anatomical data preprocessing.}

Preprocessing was performed using fMRIPrep 1.5.0 2,3 (RRID:SCR_016216), which is based on Nipype 1.2.2.5 (RRID:SCR_002502). The T1-weighted (T1w) image was corrected for intensity nonuniformity (INU) with N4BiasFieldCorrection ${ }^{6}$ (ANTs 2.2.0 $0^{7}$, RRID:SCR_004757), and used as the T1wreference throughout the workflow. The T1w-reference was skull-stripped with the antsBrainExtraction.sh workflow (ANTs) in Nipype, using OASIS30ANTs as target template. Brain tissue segmentation of cerebrospinal fluid (CSF), white-matter (WM) and gray-matter (GM) was performed on the brain-extracted T1w using fast ${ }^{8}$ (FSL 5.0.9, RRID:SCR_002823). Volume-based spatial normalization to one standard space (MNI152NLin2009cAsym) was performed through nonlinear registration with antsRegistration (ANTs 2.2.0), using brain-extracted versions of both T1w reference and the T1w template. ICBM 152 Nonlinear Asymmetrical template version $2009 c^{9}$ (RRID:SCR_008796; TemplateFlow ID: MNI152NLin2009cAsym) was used for spatial normalization.

\section{Functional data preprocessing.}

For each of the 8 BOLD scans per participant, the following preprocessing was performed. First, a reference volume and its skull-stripped version were generated using fMRIPrep. A deformation field to correct for 
susceptibility distortions was estimated based on two echo-planar imaging (EPI) references with opposing phase-encoding directions, using $3 \mathrm{dQwarp}, \mathrm{AFNI}^{10}$. Based on the estimated susceptibility distortion, an unwarped BOLD reference was calculated for a more accurate co-registration with the anatomical reference.

568 The BOLD reference was then co-registered to the T1w reference using bbregister (FreeSurfer) which implements boundary-based registration ${ }^{11}$. Co-registration was configured with six degrees of freedom. Head-motion parameters with respect to the BOLD reference (transformation matrices, and six corresponding rotation and translation parameters) were estimated before any spatiotemporal filtering using mcflirt FSL 5.0.9 ${ }^{12}$. BOLD scans were slice-time corrected using 3dTshift AFNI ${ }^{10}$ (RRID:SCR_005927). The BOLD time-series (including slice-timing correction when applied) were resampled onto their original, native space by applying a single, composite transform to correct for head-motion and susceptibility distortions. Framewise displacement (FD) confounding time-series were calculated based on the resampled BOLD time-series for each functional $\operatorname{scan}^{13}$.

\section{fMRI first-level general linear model (GLM) analyses.}

After $f M R I P r e p$ preprocessing, the first 5 volumes (10 s) of each functional scan were discarded. Then, the brain mask generated by fMRIPrep from the T1 anatomical image was used to perform brain extraction for each of the 8 functional scans. Each functional scan was then median centered. For the 6 scans of the scene exposure phase and 2 scans of the object exposure phase, all first level GLMs were performed in participants' native space with FSL using a Double-Gamma HRF with temporal derivatives, implemented with Nipype. GLMs were calculated using a variation of the Least Squares - Separate method $^{14}$ : a separate GLM was calculated for each of the 36 scenes (for scene exposure phases) or objects (for object exposure phases) across both repeats within a scan. For each GLM, there was one regressor of interest (representing a single scene or object image across its two repetitions per scan). All other trials (including lure images), framewise displacement, xyz translation and xyz rotation were represented with nuisance regressors. Additionally, a high pass filter $(128 \mathrm{~Hz})$ was applied for each GLM. This model resulted in 36 beta-maps per scan (one map per scene/object) which were converted to $t$-maps that represented the pattern of activity elicited by each scene/object for each scan.

\section{Regions of interest.}

A region of interest (ROI) for early visual cortex (EVC) was created from the probabilistic maps of Visual Topography ${ }^{15}$ in the MNI space with a 0.5 threshold. This ROI was transformed into each participant's native space using inverse T1w-to-MNI non-linear transformation. For each participant, the top 300 EVC voxels were then selected by averaging the $t$-maps of all scenes and objects and then choosing the voxels with the highest $t$-statistics (i.e., the voxels most responsive to visual stimuli). An ROI for the parahippocampal place area (PPA) was created by first using an automated meta-analysis in Neurosynth with the key term "place". Then, clusters were created using voxels with a z-score $>2$ based on the Neurosynth associative tests. Since these clusters were generated through an automated meta-analysis and were not anatomically exclusive to PPA, we visually inspected the results and manually selected the two largest clusters that were spatially consistent with PPA. One cluster was in the right hemisphere (voxel size $=247$ ) and one cluster was in the left hemisphere (voxel size $=163$ ). These clusters were combined into a single PPA mask. This mask was then transformed into each participant's native space using the inverse T1w-to-MNI transformation. For each participant, a final PPA ROI was generated by averaging the $t$-maps of all scene exposure phase scans and then selecting the 300 voxels with the highest average $t$ statistics (i.e., the most scene-responsive voxels). To create hippocampal ROIs, we used the Automatic Segmentation of Hippocampal Subfields (ASHS) ${ }^{16}$ toolbox with the upenn2017 atlas to generate subfield ROls in each participant's hippocampal body, including CA23DG - the combination of CA2, CA3 and dentate gyrus - and CA1. The most anterior and posterior slices of the hippocampal body were manually 
determined for each participant based on the T2-weighted anatomical structure. Each participant's subfield segmentations were also manually inspected to ensure accuracy of the segmentation protocol. Then, each subfield ROI was transformed into each participant's native space using the T2-to-T1w transformation, calculated with FLIRT (fsl) with 6 degrees of freedom, implemented with Nipype. All ROls were again visually inspected following the transformation to native space to ensure the ROls were anatomically correct.

\section{fMRI pattern similarity analyses.}

Pairmate Similarity Scores. Pattern similarity was calculated as the Fisher $z$-transformed Pearson correlation between $t$-maps within each ROI. All pattern similarity analyses were performed by correlating the $t$-maps for stimuli across scans (i.e., correlations were never performed within the same scan). For our primary analyses related to pattern similarity between scene images, of critical interest was mean similarity between pairmate scenes (pairmate similarity) relative to mean similarity between non-pairmate scenes (non-pairmate similarity). For example, the correlation between the $t$-maps for 'barn 1 ' from scan 3 and 'barn 2' from scan 4 would reflect pairmate similarity, whereas the correlation between the $t$-maps for 'barn 1 ' from scan 3 and 'airplane 2' from scan 4 would reflect non-pairmate similarity. We then calculated the mean difference between pairmate similarity and non-pairmate similarity, which we refer to as the pairmate similarity score.

Learned Round. To relate pairmate similarity scores to behavioral measures of learning, we identified the Learned Round (LR) for each pairmate, separately for each participant. The LR was based on performance in the associative memory test. Specifically, the LR was defined as the first round in which the target object was selected with high confidence for both scenes in a pairmate, with the additional requirement that performance remained stable in all subsequent rounds. It was therefore possible that both scenes in a pairmate were associated with high confidence correct responses in round $\mathrm{N}$, not in round $\mathrm{N}+1$, and then (again) in round $\mathrm{N}+2$ and thereafter; in this case, the LR would be round $\mathrm{N}+2$.

Inflection Point. The inflection point (IP) was defined as the transition from LR - 1 to LR (i.e., the transition from 'pre-learned' to 'learned'). Thus, pattern similarity analyses of the IP refer to the correlation of $t$-maps from LR-1 to $t$-maps from LR. We hypothesized that the behavioral state change from LR-1 to LR would correspond to a reduction in pattern similarity between pairmates. Pattern similarity analyses at the IP were contrasted against the 'pre-IP' state, which was based on the correlation of $t$-maps from LR-2 and LR-1 (i.e., a non-transition from 'not learned' to 'not learned') (Fig. 2c). Pairmates for which participants never reached and sustained high-confidence correct responses (mean \pm s.d., $1.81 \pm 2.27$ per participant) and pairmates that were learned in the $1^{\text {st }}$ round $(L R=1$; mean \pm s.d., $1.00 \pm 1.26)$ were excluded from the IP analysis because neither the pre-IP nor IP states could be measured. For pairmates that were learned in the $2^{\text {nd }}$ round ( $L R=2$; mean \pm s.d., $3.23 \pm 2.80$ ), pattern similarity at the IP was calculated and included in the analyses, but pattern similarity at the pre-IP state could not be calculated because an LR -2 did not exist. For rest of the pairmates $(\mathrm{LR}=3,4,5$, or 6$)$, we calculated pattern similarity for both pre-IP and IP (Fig. 1e). Similar restrictions applied to correlations between LR and LR-3, LR $+1, \mathrm{LR}+2$, and LR +3 (Fig. 2e). The number of pairmates included in each comparison and for each participant are reported in Supplementary Table 1.

Representational Structure Across Time Points. To test whether representational overlap triggered remapping (related to Fig. 3), the 6 learning rounds were translated into 5 timepoints. Each timepoint corresponded to a pair of consecutive learning rounds ([1,2], [2,3], [3,4], [4,5], [5,6]). For each timepoint, pairmate similarity scores were calculated, as described above, by correlating activity patterns from consecutive learning rounds (e.g., pairmate similarity scores at timepoint 1 were based on correlations 
between round 1 and round 2). This yielded a set of pairmate similarity scores at each of the 5 timepoints. These sets of similarity scores reflected the representational structure at each timepoint (i.e., which pairmates were relatively similar and which pairmates were relatively dissimilar). Pairmate similarity scores were then correlated across timepoints using Spearman's rank correlation (Fisher $z$ transformed). Lag 1 correlations refer to rank correlations between successive timepoints whereas lag 2 correlations refer to correlations between timepoints two steps apart. To facilitate a direct comparison between lag 1 vs. lag 2 correlations, correlations were computed for the following timepoints: Lag $1=r$ (timepoint 1,2$), r($ timepoint 2, 3), $r($ timepoint 3, 4); Lag $2=r($ timepoint 1, 3), $r($ timepoint 2, 4), $r$ (timepoint 3, 5). It is important to emphasize that we did not correlate initial pairmate similarity scores with the change in pairmate similarity as this would produce an artifactual correlation (via regression to the mean). In contrast, a negative rank correlation (as we observed in CA23DG) cannot be explained by regression to the mean. Mathematically, if all values at timepoint $\mathrm{N}$ partially regressed toward the mean at timepoint $\mathrm{N}+1$, this would yield a positive rank correlation (i.e., representational structure would be partially preserved). If all values fully regressed toward the mean (i.e., variance at timepoint $\mathrm{N}+1=0)$, this would yield a null correlation $(r=0$; representational structure fully abolished).

Scene-Object Similarity. To calculate pattern similarity between scenes and objects (related to Fig. 4), activation patterns for objects were first generated by averaging $t$-maps across the two object exposure phases, resulting in a single, mean activity pattern for each object. These object-specific activity patterns were then correlated with activity patterns from the scene exposure phases at LR - 1 (i.e., the pre-learned state) and LR (i.e., the learned state). Correlations were separated into three groups: (1) target correlations refer to the correlation between a scene and the object it was associated with during the study phase (e.g., 'barn 1' and 'guitar 1'), (2) competitor correlations refer to the correlation between a scene and the object that was associated with that scene's pairmate during the study phase (e.g., 'barn 1' and 'guitar 2'), and (3) across pairmate correlations refer to correlations between a scene and an object that was not associated with that scene or its pairmate during the study phase (e.g., 'barn 1' and 'scissors 1'). Target and competitor correlations were expressed relative to across pairmate correlations.

\section{Statistics.}

To compare pairmate similarity scores and other measures across ROIs and learning states, repeated measures ANOVAs and paired-samples $t$-tests were used. To test whether pairmate similarity scores and other measures were significantly positive or negative (i.e., above/below 0 ), one-sample $t$-tests were used. To test whether the negative pairmate similarity score observed in CA23DG at the inflection point depended on the specific mapping between behavioral and fMRI measures, we randomly shuffled the mapping between the behavioral inflection point and scene pairmate, within each participant (see Fig. 1d), and then computed the group-level mean pairmate similarity score at the permuted inflection point. This was repeated 1,000 times, producing a distribution of 1,000 permuted means. The observed pairmate similarity score at the inflection point was then compared against this distribution of permuted means.

\section{Data Availability.}

The data that support the findings of this study are available from the corresponding author upon reasonable request. 


\section{METHODS REFERENCES:}

702 1. Peirce, J. et al. PsychoPy2: Experiments in behavior made easy. Behav. Res. Methods 51, 195-203 703 (2019).

704 2. Esteban, O. et al. fMRIPrep: a robust preprocessing pipeline for functional MRI. Nat. Methods 16, 111$705116(2019)$.

3. Esteban, Oscar, Ross Blair, Christopher J. Markiewicz, Shoshana L. Berleant, Craig Moodie, Feilong Ma, Ayse Ilkay Isik, et al. 2018. "FMRIPrep." Software. Zenodo. https://doi.org/10.5281/zenodo.852659.

4. Gorgolewski, K. et al. Nipype: A Flexible, Lightweight and Extensible Neuroimaging Data Processing Framework in Python. Front. Neuroinformatics 5, (2011).

712 Michael Philipp Notter, Dorota Jarecka, et al. 2018. "Nipype." Software.

713 Zenodo. https://doi.org/10.5281/zenodo.596855.

714 6. Tustison, N. J. et al. N4ITK: Improved N3 Bias Correction. IEEE Trans. Med. Imaging 29, 1310-1320 715 (2010).

716 7. Avants, B. B., Epstein, C. L., Grossman, M. \& Gee, J. C. Symmetric diffeomorphic image registration 717 with cross-correlation: Evaluating automated labeling of elderly and neurodegenerative brain. Med. 718 Image Anal. 12, 26-41 (2008).

719 8. Zhang, Y., Brady, M. \& Smith, S. Segmentation of brain MR images through a hidden Markov random 720 field model and the expectation-maximization algorithm. IEEE Trans. Med. Imaging 20, 45-57 (2001).

721 9. Fonov, V., Evans, A., McKinstry, R., Almli, C. \& Collins, D. Unbiased nonlinear average age722 appropriate brain templates from birth to adulthood. Neurolmage 47, S102 (2009).

723 10. Cox, R. W. \& Hyde, J. S. Software tools for analysis and visualization of fMRI data. NMR Biomed. $724 \quad 10,171-178(1997)$.

725 11. Greve, D. N. \& Fischl, B. Accurate and robust brain image alignment using boundary-based 726 registration. Neurolmage 48, 63-72 (2009). 
727 12. Jenkinson, M., Bannister, P., Brady, M. \& Smith, S. Improved Optimization for the Robust and 728 Accurate Linear Registration and Motion Correction of Brain Images. Neurolmage 17, 825-841 (2002).

729 13. Power, J. D. et al. Methods to detect, characterize, and remove motion artifact in resting state $730 \quad$ fMRI. Neurolmage 84, 320-341 (2014).

731 14. Mumford, J. A., Turner, B. O., Ashby, F. G. \& Poldrack, R. A. Deconvolving BOLD activation in 732 event-related designs for multivoxel pattern classification analyses. Neurolmage 59, 2636-2643 733 (2012).

734 15. Wang, L., Mruczek, R. E. B., Arcaro, M. J. \& Kastner, S. Probabilistic Maps of Visual Topography 735 in Human Cortex. Cereb. Cortex N. Y. N 1991 25, 3911-3931 (2015).

736 16. Yushkevich, P. A. et al. Automated volumetry and regional thickness analysis of hippocampal 737 subfields and medial temporal cortical structures in mild cognitive impairment. Hum. Brain Mapp. 36, $738 \quad 258-287(2015)$. 
Supplementary information

\begin{tabular}{|c|c|c|c|c|c|c|c|}
\hline Participant \# & 1 & 2 & 3 & 4 & 5 & 6 & $\begin{array}{l}\text { Never } \\
\text { Learned }\end{array}$ \\
\hline 1 & 1 & 7 & 6 & 4 & 0 & 0 & 0 \\
\hline 2 & 1 & 1 & 4 & 4 & 6 & 2 & 0 \\
\hline 3 & 1 & 7 & 5 & 5 & 0 & 0 & 0 \\
\hline 4 & 0 & 3 & 0 & 5 & 4 & 3 & 3 \\
\hline 5 & 0 & 2 & 3 & 6 & 4 & 2 & 1 \\
\hline 6 & 3 & 6 & 2 & 6 & 0 & 1 & 0 \\
\hline 7 & 0 & 6 & 4 & 3 & 3 & 1 & 1 \\
\hline 8 & 0 & 2 & 5 & 4 & 5 & 1 & 1 \\
\hline 9 & 0 & 1 & 1 & 2 & 2 & 2 & 10 \\
\hline 10 & 0 & 0 & 8 & 2 & 5 & 2 & 1 \\
\hline 11 & 3 & 3 & 4 & 3 & 2 & 2 & 1 \\
\hline 12 & 0 & 1 & 2 & 5 & 2 & 5 & 3 \\
\hline 13 & 1 & 1 & 2 & 4 & 7 & 2 & 1 \\
\hline 14 & 0 & 0 & 3 & 4 & 4 & 5 & 2 \\
\hline 15 & 1 & 6 & 7 & 2 & 1 & 1 & 0 \\
\hline 16 & 1 & 2 & 6 & 1 & 2 & 4 & 2 \\
\hline 17 & 2 & 3 & 3 & 5 & 3 & 2 & 0 \\
\hline 18 & 5 & 3 & 2 & 3 & 4 & 0 & 1 \\
\hline 19 & 0 & 0 & 2 & 7 & 6 & 2 & 1 \\
\hline 20 & 0 & 1 & 6 & 2 & 1 & 4 & 4 \\
\hline 21 & 0 & 1 & 3 & 3 & 4 & 7 & 0 \\
\hline 22 & 1 & 3 & 4 & 2 & 3 & 1 & 4 \\
\hline 23 & 0 & 6 & 5 & 4 & 1 & 2 & 0 \\
\hline 24 & 3 & 4 & 7 & 1 & 2 & 1 & 0 \\
\hline 25 & 1 & 10 & 4 & 3 & 0 & 0 & 0 \\
\hline 26 & 0 & 0 & 2 & 9 & 2 & 1 & 4 \\
\hline 27 & 3 & 0 & 4 & 2 & 2 & 1 & 6 \\
\hline 28 & 1 & 8 & 4 & 3 & 0 & 0 & 2 \\
\hline 29 & 0 & 6 & 2 & 1 & 1 & 2 & 6 \\
\hline 30 & 2 & 6 & 6 & 1 & 0 & 2 & 1 \\
\hline 31 & 1 & 1 & 3 & 6 & 3 & 3 & 1 \\
\hline
\end{tabular}

Table1. Number of pairmates that transitioned to learned round ('LR') status, for each participant and each round. Note: pairmates that were learned in the first round or never learned were excluded from fMRI analyses. 